\title{
PTU-118 $九$ P21/CDKN1A GERMLINE VARIATION AND LOW PENETRANCE PREDISPOSITION TO COLORECTAL CANCER
}

doi:10.1136/gut.2011.239301.246

K J Monahan, ${ }^{1,2, *}$ S Spain, ${ }^{3}$ H J Thomas, ${ }^{2}$ I P Tomlinson ${ }^{3}{ }^{1}$ Gastroenterology, West Middlesex University Hospital, London, UK; ${ }^{2}$ Cancer Medicine, Imperial College, London, UK; ${ }^{3}$ Molecular and Population Genetics, Cancer Research UK, London, UK

Introduction Progressive loss of cell cycle control is an important feature on the adenoma-carcinoma sequence of colorectal cancer. Cyclin-dependent kinase inhibitor 1A (P21/CDKN1A) is an important target of the TGF $\beta$ signalling pathway, and it is commonly under-expressed as colorectal neoplasia develop. The aim of this study was to identify low penetrance germline variation in this gene which predisposes individuals to colorectal cancer.

Methods Variation in the coding region of CDKN1A was determined in fifty colorectal cancer cases with a strong family history and 50 controls were tested using the Lightscanner and direct sequencing. 15 tagging SNPs around CDKN1A were typed in the CORGI cases and controls as part of a genomewide analysis using the Illumina Hap550 platform in 930 cases and 960 controls performed by colleagues (Tomlinson et al 2007). Allele-specific expression of the gene was examined using quantitative reverse transcriptase PCR linked to SNPs in an upstream promoter region.

Results A novel amino-acid changing variant Phe22Leu was identified in a single colorectal cancer patient. Six patients were identified in the case group and 5 in the control group with Arg31Ser. In the association study the two most significant SNPs lie in an upstream promoter region and are in linkage disequilibrium, both are risk alleles for colorectal cancer (OR 1.13; 95\% CI 1.06 to 1.2). There were significant differences in expression between CDKN1A and the controls in 94 samples ( $\mathrm{p}=0.037$, Student's t test), demonstrating linkage of these upstream polymorphisms to allele-specific expression of CDKN1A.

Conclusion Rare variants of $P 21 / C D K N 1 A$ are an infrequent cause of predisposition to colorectal neoplasia. A promoter region upstream of the CDKN1A is associated with prediposition to colorectal neoplasia, and is linked to allele-specific expression of this gene.

Competing interests $\mathrm{K}$. Monahan Grant/Research

Support from: Bobby Moore Fund, S. Spain: None Declared, H. Thomas: None Declared, I. Tomlinson: None Declared.

Keywords colorectal cancer, genetics, P21, tumourigenesis. 


\section{REFERENCE}

1. Tomlinson I, Webb E, Carvajal-Carmona L, et al. A genome-wide association scan of tag SNPs identifies a susceptibility variant for colorectal cancer at 8q24.21.

Nature Genetics 2007. 